www.jmscr.igmpublication.org

Impact Factor (SJIF): 6.379

Index Copernicus Value: 71.58

ISSN (e)-2347-176x ISSN (p) 2455-0450

crossref DOI: _https://dx.doi.org/10.18535/jmscr/v6i3.139

Journal Of Medical Science And Clinical Research

\title{
A Prospective Comparative Study between Bone Biomarkers \& DEXA Scan Absorptiometry in Diagnosis \& Therapeutic post treatment follow up of Osteoporosis
}

\section{Authors \\ Ahmed Alkhuzai FICMS, Hisham A. Getta FICMS, Salah M. Fateh DMRD/FIBMS}

Faculty of Medicine, Sulaimani University, Iraq

\begin{abstract}
Introduction: We try to study the best method in diagnosis \& therapeutic follow up post treatment respond in osteoporotic patients. Used the advantages of Dexa scan Absorptiometry and bone biomarkers for diagnosis \& therapeutics post treatment follow up monitoring of osteoporotic patients were treated by different modalities.

Methods: Prospective comparative studies were randomized according to national osteoporotic foundation. Performed on 200 osteoporotic patients from March 2010 to April 2012, in private clinic sharing private Hematological and Dexa Scan Absorptiometry clinic in Sulaimaniyah city. All these patients with clinical sign of osteoporosis, also all of patients with history of osteoporotic fractures plus surgical history "Hysterectomy, Mastectomy, ooperectomy" were classified into two groups. Group A 100 patients used Dexa scan Absorptiometry for diagnosis \& therapeutics treatment monitoring follow up. Group B100 patients were used bone biomarker "Vit D3, PTH, ALK, Osteocalcin" for evaluation \& monitoring bone turnover used in diagnosis \& therapeutic post treatment follow up of osteoporotic patients. The patients were follow up for 2 years period. The outcome measurements were according to Ancillary results scores of national osteoporotic foundations.

Results: In both group A \& group B significant reduction in the mean of Ancillary results scores base line for both Dexa scan Absorptiometry \& bone biomarkers were seen early \& at 6 months, 1 year and 2 years follow up period visit of osteoporotic patients. Also, there were significant differences ( $p$-value < $0.0001)$ to the same period of follow up between groups A there were used Dexa scan Absorptiometry as superior to bone biomarkers in diagnosis of osteoporotic patients. In Compared to group B depend on bone biomarkers there were superior in therapeutics post treatment follow up than Dexa scan Absorptiometry.

Conclusions: We concluded that Dexa scan Absorptiometry \& Bone Biomarkers both are significant in diagnosis and post therapeutic treatment follow up of osteoporotic patients, However, Bone Biomarkersis superior in post therapeutic treatment than Dexa Scan Absorptiometry which is superior in diagnosis of osteoporotic patients for 2 years study follow up.
\end{abstract}

\section{Introduction}

Osteoporosis is a silent disease until it is complicated by fractures that can occur following minimal trauma. The disease can be prevented, diagnosed and treated before any fracture occurs. Prevention, detection and treatment of 
osteoporosis should be a mandate of primary care providers $^{1}$. Osteoporosis is second only to cardiovascular disease as a leading health care problem, according to the World Health Organization. Worldwide, the lifetime risk for women to have an osteoporotic fracture is 30$40 \%{ }^{2}$. Because of related morbidity, disability, diminished quality of life, and mortality, osteoporosis and fractures associated with it are major public health concern ${ }^{3}$. Since the National Osteoporosis Foundation NOF first published the Guide in 1999, it has become increasingly clear that many patients are not being given appropriate information about prevention; too many patients are not being prescribed any of the FDAapproved, effective therapies. This Guide offers concise recommendations regarding prevention, risk assessment, diagnosis and treatment of osteoporosis in postmenopausal women and men age 50 and older. It includes indications for bone densitometry and fracture risk thresholds for intervention with pharmacologic agents ${ }^{1}$. Dualenergy $\mathrm{x}$-ray Absorptiometry (DXA) measurement of the hip and spine is the technology now used to establish or confirm a diagnosis of osteoporosis, predict future fracture risk and monitor patients by performing serial assessments ${ }^{4}$ Bone Biomarkers are released into the circulation during the process of bone formation and resorption, providing information about the dynamic process of bone metabolism. During bone remodeling, bone formation by osteoblasts and bone resorption by osteoclasts are tightly coupled in time and space within the bone multicellular unit ${ }^{5}$. Drugs that promote bone formation (e.g. parathyroid hormone) increase biomarkers of formation and resorption, and drugs that inhibit resorption (e.g. Bisphosphonates) decrease biomarkers of formation and resorption ${ }^{6}$. Biomarkers of bone turnover predict fractures and changes in bone mineral density in adults, and can be used to monitor the effectiveness of therapy ${ }^{7,8}$. Numerous studies have demonstrated good correlations between biomarkers of bone metabolism and the actual rate of bone turnover, as quantified by calcium kinetics studies ${ }^{9}$, or by histomorphometric analyses of labeled bone biopsies $^{10}$. Although biochemical markers of bone turnover may be a more sensitive way of monitoring bone response, their use is subject to the previously noted limitations. Nevertheless, decreases in bone resorption markers measured 3 to 6 months after initiating antiresorptive therapy or increases in formation markers 1 to 3 months after starting anabolic therapy predict the subsequent increases in BMD and reduction in fracture rates and make them an attractive consideration as part of the clinical management of pharmacologic therapy ${ }^{11}$. Osteocalcin (OC), a bone-specific protein synthesized by the osteoblasts in bone, is the major non-collagen protein in the bone matrix. It has a molecular weight of 5,800 Da and contains 49 amino acids, including 3 gamma carboxyl glutamic acid residues that facilitate the binding of $\mathrm{OC}$ to hydroxyapatite in bone ${ }^{12}$. The serum OC level, a sensitive marker of bone production, is associated with a high bone turnover rate and decreased $\mathrm{BMD}$, and correlates well with histomorphometric indices of bone formation ${ }^{13}$.

Bone Markers of bone turnover are considered useful diagnostic tools for the evaluation of bone formation and resorption. We measured serum OC and total serum alkaline phosphatase (ALP) activity as markers of bone formation. The objectives of this study were to compare between these bone marrow markers and Dexa Scan in diagnosis and therapeutic follow up for predicting the osteoporosis by taking into consideration age, body mass index (BMI), and menopausal status. What are Bone Biomarkers: bone biomarker "Vit D3, PTH, ALK, C-Terminal Telopeptides, Osteocalcin" for evaluation \& monitoring bone formation and resorption turnover in the blood as indicators for the osteoporosis level in the body. 


\section{JMSCR Vol||06||Issue||03||Page 827-840||March}
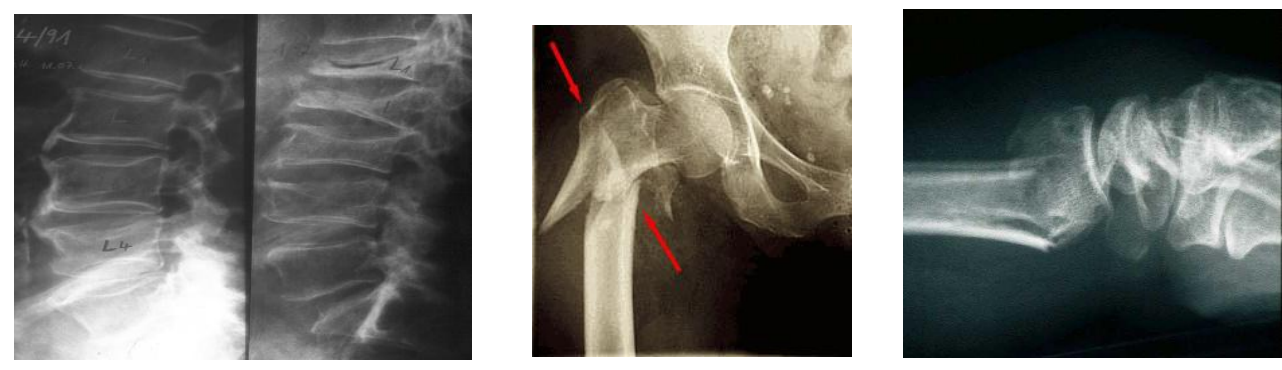

Fig.:1 Osteoporosis Fractures

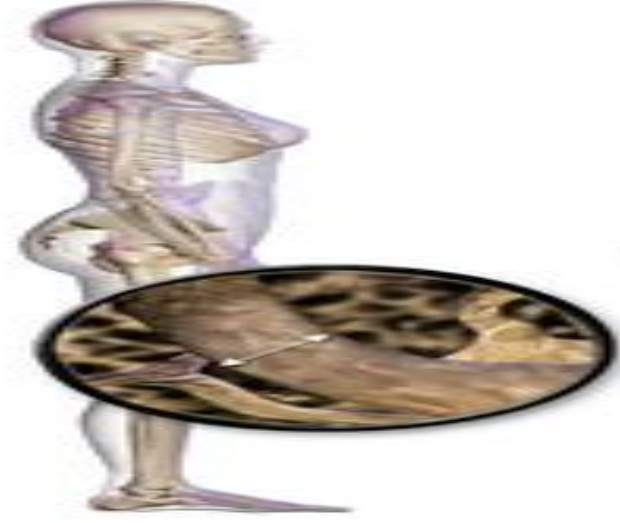

Fig.2: Before Metabolic changes

\section{Patients \& Method}

This is a prospective comparative study in Sulaimaniyah private clinic, to compare the dual X-ray Absorptiometry (DXA) scan and Bone Biomarkers in detection the most sensitive method for early diagnosis plus therapeutic post treatment follow up of osteoporosis. The bone Biomarkers include serum Osteocalcin, ALK bone specific alkaline phosphatase, Vit D3, PTH parathyroid hormonal assessments. For evaluation with monitoring bone turnover used in for both purposes of diagnosis \& therapeutic post treatment follow up of osteoporotic patients, at early date, then at 6 months, 12 months, and 24 months. Were randomized according to NOF National Osteoporotic Foundation, performed on 200 osteoporotic patients from March 2010 to April 2012, were received in private orthopedic clinic sharing with private hematological and Dexa Scan Absorptiometry clinic in Sulaimaniyah city. All these patients with clinical sign of osteoporosis, also all of these patients with history of osteoporotic fractures plus surgical history "Hysterectomy, Mastectomy, ooperectomy" were classified into two groups. Group A 100 patients

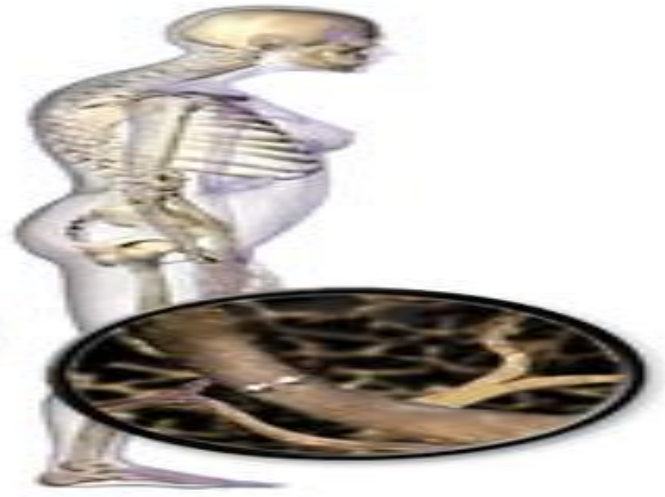

After Metabolic changes

used Dexa scan Absorptiometry for diagnosis monitoring osteoporotic patients.

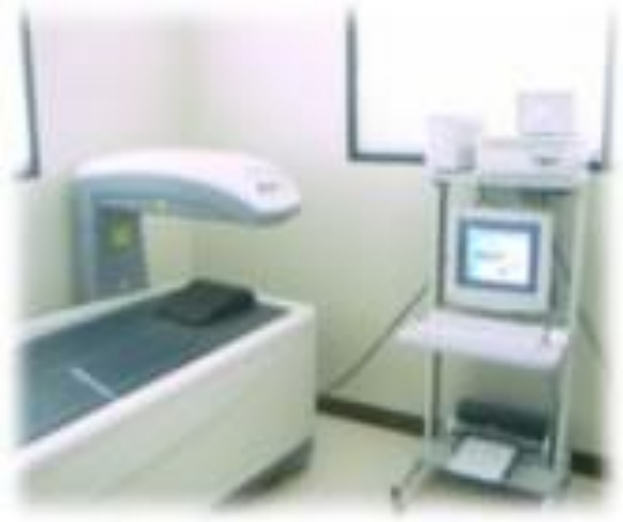

Fig 3: Dexa Scan Absorptiometry for Scanning the osteoporotic patients

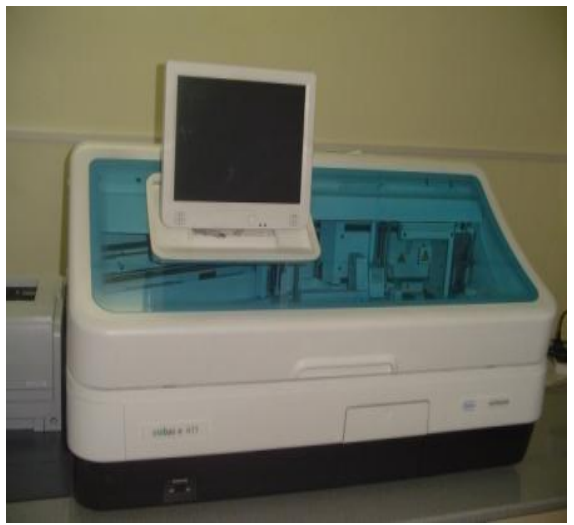

Fig 4: Casa Bone Biomarkers Assessment 
Table 1: WHO Definition of Osteoporosis Based on BMD

\begin{tabular}{|l|c|c|}
\hline T-score & BMD & \\
\hline T-score at -1.0 and above & $\begin{array}{c}\text { Within 1 SD of a young-adult } \\
\text { reference population }\end{array}$ & Normal \\
\hline $\begin{array}{l}\text { T-score between -1.0 and - } 2.5 \\
\text { Between 1.0 and 2.5 SD below } \\
\text { that of a young-adult reference } \\
\text { population }\end{array}$ & Low Bone Mass (Osteopenia) \\
\hline T-score at or below -2.5 & $\begin{array}{c}2.5 \text { SD or more below that of a } \\
\text { young- adult reference population }\end{array}$ & Osteoporosis \\
\hline $\begin{array}{l}\text { T-score at or below }-2.5 \text { with } \\
\text { one or more fractures }\end{array}$ & $\begin{array}{c}2.5 \text { SD or more below that of a } \\
\text { young- adult reference population }\end{array}$ & $\begin{array}{c}\text { Severe or Established } \\
\text { Osteoporosis }\end{array}$ \\
\hline
\end{tabular}

Group B100 patients were used bone biomarker "Vit D3, PTH, ALK, Osteocalcin" for evaluation \& monitoring bone formation and resorption turnover used both in diagnosis \& therapeutic post treatment follow up of osteoporosis. The patients were following up for 2 years period, the outcome measurements were according to Ancillary results scores of national osteoporotic foundations.

T score - This number shows the amount of bone have compared with a young adult of the same gender with peak bone mass. A score above -1 is considered normal. A score between -1 and 2.5 is classified as osteopenia (low bone mass). A score below -2.5 is defined as osteoporosis. The $\mathrm{T}$ score is used to estimate the risk of developing fracture.

\section{Results}

We detected the early in diagnosis of 100 patients, the used Dexa Scan Absorptiometry there were $100 \%$ diagnosed as Osteoporosis, $85 \%$ diagnosis by Bone Biomarkers. However, 78\% data changes were detected by Bone Biomarkers in compared to $33 \%$ detected by Dexa Scan Absorptiometry in post therapeutic treatments follow up osteoporotic patients after $24^{\text {th }}$ months. Also, we observed the Group A \& Group B has significant reduction in the mean of Ancillary results scores from base line for both Dexa scan Absorptiometry \& bone biomarkers were seen early and at 6, 12, and 24 months follow up period visit of osteoporotic patients. Also, there were significant differences ( $\mathrm{p}$-value $<0.0001$ ) to the same period of follow up between groups A there were used Dexa scan Absorptiometry as superior to bone biomarkers in early visit for diagnosis of osteoporotic patients. In Compared to group B were depend on bone Biomarkers alone, there were superior in therapeutics post treatment follow up than the Dexa scan Absorptiometry observed by law rate of change at 6 months, then improved at 12 months, with return to normal in 24 months, while Dexa Scan Absorptiometry has little or no changes during same period of follow up. The high sensitive index of Biomarkers detection during follow up assessment at $6^{\text {th }}, 12^{\text {th }}, 24^{\text {th }}$ months as dependents data assessment of osteoporotic patients. As shown in the tables; 
Table 2: Early in diagnosis

\begin{tabular}{|c|c|c|c|c|c|c|c|c|}
\hline \multirow[t]{2}{*}{ No } & \multirow[t]{2}{*}{ Age } & \multirow[t]{2}{*}{ Sex } & \multirow[t]{2}{*}{$\begin{array}{l}\text { Time of presentation } \\
\text { (early in diagnosis) }\end{array}$} & \multicolumn{3}{|c|}{ Biomarkers } & \multicolumn{2}{|c|}{$\begin{array}{c}\text { BMD } \\
\text { Scan / T Score }\end{array}$} \\
\hline & & & & PTH & VIT D & Osteocalcin & Lumbar & Femur \\
\hline 1 & 52 & $\mathbf{F}$ & Early in diagnosis & 66.24 & 5.3 & 12.75 & -2.8 & -2.6 \\
\hline 2 & 54 & $\mathbf{F}$ & Early in diagnosis & 83.11 & 3.34 & 23.89 & -2.5 & -2.7 \\
\hline 3 & 56 & $\mathbf{F}$ & Early in diagnosis & 66.17 & 9.82 & 12.35 & -4.2 & -3.9 \\
\hline 4 & 53 & $\mathbf{F}$ & Early in diagnosis & 72.1 & 8.69 & 14.69 & -3.5 & -3.6 \\
\hline 5 & 77 & $\mathbf{M}$ & Early in diagnosis & 83.45 & 7.99 & 17.14 & -2.8 & -2.8 \\
\hline 6 & 82 & $\mathbf{F}$ & Early in diagnosis & 99.95 & 8.75 & 34.54 & -2.6 & -2.5 \\
\hline 7 & 66 & $\mathbf{F}$ & Early in diagnosis & 66.16 & 9.14 & 25.38 & -3.8 & -4.4 \\
\hline 8 & 63 & $\mathbf{F}$ & Early in diagnosis & 93.96 & $\mathbf{4 . 5 3}$ & 32.64 & -3.1 & -3.3 \\
\hline 9 & 76 & $\mathbf{F}$ & Early in diagnosis & 79.39 & 2.97 & 37.2 & -4.9 & -3.9 \\
\hline 10 & 83 & $\mathbf{M}$ & Early in diagnosis & 80.28 & 3.2 & 11.77 & -4.6 & -4.8 \\
\hline 11 & 79 & $\mathbf{M}$ & Early in diagnosis & 66.2 & 9.56 & 20.06 & -3.1 & -3.0 \\
\hline 12 & 59 & $\mathbf{F}$ & Early in diagnosis & 88.46 & 8.25 & 20.54 & -4.0 & -4.5 \\
\hline 13 & 56 & $\mathbf{F}$ & Early in diagnosis & 88.7 & 6.46 & 57 & -3.2 & -3.1 \\
\hline 14 & 62 & $\mathbf{F}$ & Early in diagnosis & 79.15 & 8.64 & 17.94 & -3.9 & -3.5 \\
\hline 15 & 64 & $\mathbf{F}$ & Early in diagnosis & 79.58 & $<3$ & 6.31 & -4.2 & -4.0 \\
\hline 16 & 74 & $\mathbf{F}$ & Early in diagnosis & 99.05 & 2.46 & 141.3 & -4.3 & -4.7 \\
\hline 17 & 53 & $\mathbf{F}$ & Early in diagnosis & 407.7 & 6.1 & 75.66 & -3.2 & -2.9 \\
\hline 18 & 48 & $\mathbf{F}$ & Early in diagnosis & 74.82 & 7.72 & 37.31 & -3.9 & -2.9 \\
\hline 19 & 57 & $\mathbf{F}$ & Early in diagnosis & 85.91 & 6.06 & 11.08 & -3.8 & -3.6 \\
\hline 20 & 46 & $\mathbf{M}$ & Early in diagnosis & 65.87 & 9.17 & 22.62 & -2.9 & -2.7 \\
\hline 21 & 49 & $\mathbf{F}$ & Early in diagnosis & 97.06 & 2.09 & 10.34 & -3.8 & -4.2 \\
\hline 22 & 45 & $\mathbf{F}$ & Early in diagnosis & 77.25 & 3.27 & 7.77 & -2.6 & -2.8 \\
\hline 23 & 65 & $\mathbf{F}$ & Early in diagnosis & 86.69 & 4.04 & 23.65 & -3.8 & -4.1 \\
\hline 24 & 64 & $\mathbf{F}$ & Early in diagnosis & 82.86 & 6.56 & 22.34 & -3.7 & -3.4 \\
\hline 25 & 68 & $\mathbf{F}$ & Early in diagnosis & 85.83 & 1.73 & 56.87 & -4.5 & -3.9 \\
\hline 26 & 88 & $\mathbf{F}$ & Early in diagnosis & 98.3 & 4.94 & 25.45 & -3.9 & -4.0 \\
\hline 27 & 74 & $\mathbf{F}$ & Early in diagnosis & 74.15 & 4.79 & 32.23 & -3.6 & -3.8 \\
\hline 28 & 86 & $\mathbf{F}$ & Early in diagnosis & 84.48 & 6.87 & 19.94 & -4.9 & -4.7 \\
\hline 29 & 77 & $\mathbf{F}$ & Early in diagnosis & 292.3 & 3.84 & 90.91 & -3.5 & -3.3 \\
\hline 30 & 80 & $\mathbf{M}$ & Early in diagnosis & 90.2 & 2.22 & 22.5 & -3.8 & -3.4 \\
\hline 31 & 78 & $\mathbf{F}$ & Early in diagnosis & 76.7 & 4.56 & 20.33 & -3.4 & -3.6 \\
\hline 32 & 69 & $\mathbf{F}$ & Early in diagnosis & 88.2 & 6.05 & 21.98 & -3.2 & -3.5 \\
\hline 33 & 46 & $\mathbf{F}$ & Early in diagnosis & 87.6 & 8.58 & 19.9 & -2.9 & -2.8 \\
\hline 34 & 47 & $\mathbf{F}$ & Early in diagnosis & 280.5 & 3.37 & 29.19 & -2.9 & -2.5 \\
\hline 35 & 78 & $\mathbf{M}$ & Early in diagnosis & 66.44 & 9.06 & 14.59 & -3.1 & -3.0 \\
\hline 36 & 44 & $\mathbf{F}$ & Early in diagnosis & 70.6 & 2.32 & 51.56 & -2.5 & -2.6 \\
\hline 37 & 49 & $\mathbf{F}$ & Early in diagnosis & 90.2 & 5.46 & 23.76 & -2.8 & -2.8 \\
\hline 38 & 52 & $\mathbf{F}$ & Early in diagnosis & 107.3 & 4.47 & 13.08 & -2.7 & -2.9 \\
\hline 39 & 65 & $\mathbf{F}$ & Early in diagnosis & 80.06 & 8.71 & 12.58 & -3.2 & -3.4 \\
\hline 40 & 81 & $\mathbf{M}$ & Early in diagnosis & 90.06 & 6.17 & 11.05 & -3.1 & -3.3 \\
\hline 41 & 64 & $\mathbf{F}$ & Early in diagnosis & 81.09 & 1.37 & 29.93 & -4.2 & -3.9 \\
\hline 42 & 81 & $\mathbf{F}$ & Early in diagnosis & 67.02 & 9.56 & 29.66 & -4.3 & -4.6 \\
\hline 43 & 78 & $\mathbf{F}$ & Early in diagnosis & 68.06 & 1.08 & 22.06 & -3.8 & -4.4 \\
\hline 44 & 83 & $\mathbf{M}$ & Early in diagnosis & 103.5 & 7.69 & 29.36 & -4.0 & -3.6 \\
\hline 45 & 82 & $\mathbf{M}$ & Early in diagnosis & 74.08 & 2.33 & 24.25 & -3.8 & -3.9 \\
\hline 46 & 76 & $\mathbf{F}$ & Early in diagnosis & 70.01 & 9.22 & 20.06 & -3.7 & -3.7 \\
\hline 47 & 75 & $\mathbf{F}$ & Early in diagnosis & 77.04 & 8.35 & 41.00 & -3.3 & -3.6 \\
\hline 48 & 80 & $\mathbf{F}$ & Early in diagnosis & 109.6 & 1.49 & 33.35 & -4.1 & -4.4 \\
\hline 49 & 47 & $\mathbf{F}$ & Early in diagnosis & 85.08 & 3.99 & 19.00 & -3.3 & -2.9 \\
\hline 50 & 80 & $\mathbf{M}$ & Early in diagnosis & 140.8 & 9.85 & 38.87 & -2.8 & -2.5 \\
\hline 51 & 68 & $\mathbf{F}$ & Early in diagnosis & 82.12 & 4.2 & 14.55 & -3.6 & -4.1 \\
\hline 52 & 46 & $\mathbf{F}$ & Early in diagnosis & 66.07 & 9.38 & 25.67 & -2.8 & -2.7 \\
\hline 53 & 47 & $\mathbf{F}$ & Early in diagnosis & 99.2 & 2.27 & 16.39 & -2.9 & -2.6 \\
\hline 54 & 48 & $\mathbf{F}$ & Early in diagnosis & 88.5 & 7.49 & 17.43 & -3.0 & -2.8 \\
\hline 55 & 69 & $\mathbf{F}$ & Early in diagnosis & 68.42 & 6.88 & 13.16 & -4.0 & -3.7 \\
\hline 56 & 65 & $\mathbf{F}$ & Early in diagnosis & 93.65 & 9.55 & 31.14 & -3.9 & -4.3 \\
\hline 57 & 70 & $\mathbf{F}$ & Early in diagnosis & 73.11 & 7.19 & 22.31 & -3.8 & -3.2 \\
\hline 58 & 79 & $\mathbf{F}$ & Early in diagnosis & 86.16 & 8.24 & 36.42 & -4.1 & -4.3 \\
\hline 59 & 77 & $\mathbf{M}$ & Early in diagnosis & 75.32 & 2.87 & 31.12 & -4.2 & -3.8 \\
\hline 60 & 79 & $\mathbf{M}$ & Early in diagnosis & 88.20 & 3.4 & 16.87 & -4.5 & -4.9 \\
\hline 61 & 68 & $\mathbf{F}$ & Early in diagnosis & 95.32 & 4.51 & 30.16 & -3.8 & -3.8 \\
\hline 62 & 72 & $\mathbf{F}$ & Early in diagnosis & 85.47 & 6.35 & 26.64 & -3.3 & -3.5 \\
\hline 63 & 72 & $\mathbf{F}$ & Early in diagnosis & 65.17 & 9.91 & 53.34 & -4.0 & -3.7 \\
\hline 64 & 70 & $\mathbf{F}$ & Early in diagnosis & 79.15 & 6.24 & 13.45 & -3.4 & -3.6 \\
\hline 65 & 48 & $\mathbf{F}$ & Early in diagnosis & 66.92 & 9.88 & 6.15 & -3.1 & -2.7 \\
\hline 66 & 49 & $\mathbf{F}$ & Early in diagnosis & 85.04 & 2.42 & 15.23 & -2.9 & -3.8 \\
\hline 67 & 67 & $\mathbf{F}$ & Early in diagnosis & 407.7 & 6.1 & 75.66 & -3.3 & -3.0 \\
\hline 68 & 46 & $\mathbf{F}$ & Early in diagnosis & 66.82 & 9.72 & 37.31 & -2.6 & -2.9 \\
\hline 69 & 49 & $\mathbf{F}$ & Early in diagnosis & 75.91 & 6.06 & 11.08 & -2.8 & -2.8 \\
\hline
\end{tabular}




\begin{tabular}{|c|c|c|c|c|c|c|c|c|}
\hline 70 & 78 & M & Early in diagnosis & 68.87 & 5.7 & 22.62 & -2.8 & -2.5 \\
\hline 71 & 81 & $\mathbf{M}$ & Early in diagnosis & 107.6 & 3.19 & 16.44 & -3.5 & -3.5 \\
\hline 72 & 69 & $\mathbf{F}$ & Early in diagnosis & 77.15 & $\mathbf{3 . 3 7}$ & 17.77 & -3.2 & -4.7 \\
\hline 73 & 67 & $\mathbf{F}$ & Early in diagnosis & 88.78 & 9.07 & 21.45 & -3.8 & -3.3 \\
\hline 74 & 78 & $\mathbf{F}$ & Early in diagnosis & 80.80 & 4.56 & 26.23 & -4.5 & -4.8 \\
\hline 75 & 63 & $\mathbf{F}$ & Early in diagnosis & 89.88 & 3.79 & 59.67 & -3.2 & -3.4 \\
\hline 76 & 82 & $\mathbf{F}$ & Early in diagnosis & 67.13 & 9.79 & 23.35 & -4.5 & -4.5 \\
\hline 77 & 77 & $\mathbf{F}$ & Early in diagnosis & 66.55 & 9.84 & 38.22 & -3.2 & -3.5 \\
\hline 78 & 78 & $\mathbf{F}$ & Early in diagnosis & 71.48 & 7.87 & 14.94 & -3.3 & -3.7 \\
\hline 79 & 76 & $\mathbf{F}$ & Early in diagnosis & 277.3 & 3.66 & 90.80 & -4.2 & -3.8 \\
\hline 80 & 83 & $\mathbf{F}$ & Early in diagnosis & 98.2 & 6.32 & 42.25 & -4.6 & -4.6 \\
\hline 81 & 43 & $\mathbf{F}$ & Early in diagnosis & 76.27 & 8.56 & 28.33 & -2.8 & -2.9 \\
\hline 82 & 46 & $\mathbf{F}$ & Early in diagnosis & 67.02 & 9.05 & 27.78 & -2.7 & -3.8 \\
\hline 83 & 66 & $\mathbf{F}$ & Early in diagnosis & 73.26 & 8.68 & 39.19 & -3.0 & -3.1 \\
\hline 84 & 44 & $\mathbf{F}$ & Early in diagnosis & 230.8 & 3.67 & 27.59 & -2.7 & -2.9 \\
\hline 85 & 78 & $\mathbf{M}$ & Early in diagnosis & 87.14 & 8.16 & 16.89 & -2.5 & -2.5 \\
\hline 86 & 74 & $\mathbf{M}$ & Early in diagnosis & 74.6 & 4.42 & 61.76 & -2.8 & -3.0 \\
\hline 87 & 72 & $\mathbf{F}$ & Early in diagnosis & 80.4 & 5.86 & 33.56 & -3.2 & -2.6 \\
\hline 88 & 73 & $\mathbf{F}$ & Early in diagnosis & 102.3 & 4.67 & 19.68 & -2.7 & -3.3 \\
\hline 89 & 78 & $\mathbf{F}$ & Early in diagnosis & 77.66 & 9.81 & 22.38 & -3.9 & -4.2 \\
\hline 90 & 84 & $\mathbf{F}$ & Early in diagnosis & 66.06 & 9.17 & 14.55 & -4.0 & -4.1 \\
\hline 91 & 79 & $\mathbf{F}$ & Early in diagnosis & 88.04 & 5.87 & 27.23 & -4.2 & -3.8 \\
\hline 92 & 85 & $\mathbf{F}$ & Early in diagnosis & 78.52 & 2.57 & 25.06 & -3.3 & -4.3 \\
\hline 93 & 77 & $\mathbf{F}$ & Early in diagnosis & 82.06 & 6.08 & 26.86 & -3.6 & -3.5 \\
\hline 94 & 81 & $\mathbf{M}$ & Early in diagnosis & 113.5 & 8.67 & 26.31 & -3.8 & -3.9 \\
\hline 95 & 80 & $\mathbf{M}$ & Early in diagnosis & 78.78 & 3.53 & 34.15 & -2.5 & -3.1 \\
\hline 96 & 76 & $\mathbf{F}$ & Early in diagnosis & 78.11 & 5.62 & 28.36 & -3.8 & -3.7 \\
\hline 97 & 75 & $\mathbf{F}$ & Early in diagnosis & 94.14 & 8.55 & 44.33 & -4.5 & -3.8 \\
\hline 98 & 45 & $\mathbf{F}$ & Early in diagnosis & 129.6 & 2.44 & 34.75 & -3.9 & -4.4 \\
\hline 99 & 48 & $\mathbf{F}$ & Early in diagnosis & 72.68 & 6.89 & 29.33 & -2.8 & -2.9 \\
\hline 100 & 79 & $\mathbf{M}$ & Early in diagnosis & 134.8 & 6.25 & 27.67 & -2.6 & -2.6 \\
\hline
\end{tabular}

Table 3: 6 months post therapy

\begin{tabular}{|c|c|c|c|c|c|c|c|c|}
\hline \multirow[t]{2}{*}{ No } & \multirow[t]{2}{*}{ Age } & \multirow[t]{2}{*}{ Sex } & \multirow{2}{*}{$\begin{array}{c}\text { Time of } \\
\text { Presentation } \\
\left(6^{\text {th }} \text { months later }\right)\end{array}$} & \multicolumn{3}{|c|}{ Biomarkers } & \multicolumn{2}{|c|}{$\begin{array}{c}\text { BMD } \\
\text { Scan / T Score }\end{array}$} \\
\hline & & & & PTH & VIT D & Osteocalcin & Lumbar & Femur \\
\hline 1 & 52 & $\mathbf{F}$ & $6^{\text {th }}$ months later & 60.22 & 15.3 & 14.55 & -2.7 & -2.6 \\
\hline 2 & 54 & $\mathbf{F}$ & $6^{\text {th }}$ months later & 83.11 & 3.34 & 20.79 & -2.5 & -2.7 \\
\hline 3 & 56 & $\mathbf{F}$ & $6^{\text {th }}$ months later & 58.17 & 23.22 & 11.33 & -4.2 & -3.9 \\
\hline 4 & 53 & $\mathbf{F}$ & $6^{\text {th }}$ months later & 72.1 & 8.69 & 24.69 & -3.5 & -3.6 \\
\hline 5 & 77 & $\mathbf{M}$ & $6^{\text {th }}$ months later & 61.45 & 17.88 & 14.14 & -2.8 & -2.8 \\
\hline 6 & 82 & $\mathbf{F}$ & $6^{\text {th }}$ months later & 99.95 & 8.75 & 32.24 & -2.6 & -2.5 \\
\hline 7 & 66 & $\mathbf{F}$ & $6^{\text {th }}$ months later & 60.16 & 19.14 & 21.98 & -3.2 & -3.9 \\
\hline 8 & 63 & $\mathbf{F}$ & $6^{\text {th }}$ months later & 93.96 & 4.53 & 39.64 & -3.1 & -3.3 \\
\hline 9 & 76 & $\mathbf{F}$ & $6^{\text {th }}$ months later & 62.23 & 16.77 & 30.02 & -4.9 & -3.9 \\
\hline 10 & 83 & $\mathbf{M}$ & $6^{\text {th }}$ months later & 80.28 & 3.2 & 21.77 & -4.6 & -4.8 \\
\hline 11 & 79 & M & $6^{\text {th }}$ months later & 58.12 & 23.46 & 25.16 & -3.1 & -3.0 \\
\hline 12 & 59 & $\mathbf{F}$ & $6^{\text {th }}$ months later & 60.44 & 18.20 & 27.84 & -4.0 & -4.5 \\
\hline 13 & 56 & $\mathbf{F}$ & $6^{\text {th }}$ months later & 88.7 & 6.46 & 24.57 & -3.2 & -3.1 \\
\hline 14 & 62 & $\mathbf{F}$ & $6^{\text {th }}$ months later & 79.15 & 8.64 & 13.44 & -3.9 & -3.5 \\
\hline 15 & 64 & $\mathbf{F}$ & $6^{\text {th }}$ months later & 59.58 & 12.22 & 16.31 & -4.2 & -4.0 \\
\hline 16 & 74 & $\mathbf{F}$ & $6^{\text {th }}$ months later & 99.05 & 2.46 & 166.3 & -4.3 & -4.7 \\
\hline 17 & 53 & $\mathbf{F}$ & $6^{\text {th }}$ months later & 407.7 & 6.1 & 70.46 & -3.2 & -2.9 \\
\hline 18 & 48 & $\mathbf{F}$ & $6^{\text {th }}$ months later & 58.82 & 22.72 & 30.21 & -3.9 & -2.9 \\
\hline 19 & 57 & $\mathbf{F}$ & $6^{\text {th }}$ months later & 85.91 & 6.06 & 17.08 & -3.8 & -3.6 \\
\hline 20 & 46 & $\mathbf{M}$ & $6^{\text {th }}$ months later & 52.87 & 20.17 & 23.62 & -2.9 & -2.7 \\
\hline 21 & 49 & $\mathbf{F}$ & $6^{\text {th }}$ months later & 56.36 & 12.29 & 11.34 & -3.8 & -4.2 \\
\hline 22 & 45 & $\mathbf{F}$ & $6^{\text {th }}$ months later & 77.25 & 3.27 & 17.77 & -2.6 & -2.8 \\
\hline 23 & 65 & $\mathbf{F}$ & $6^{\text {th }}$ months later & 52.69 & 19.14 & 26.65 & $\begin{array}{ll}-3.8 \\
\end{array}$ & -4.1 \\
\hline 24 & 64 & $\mathbf{F}$ & $6^{\text {th }}$ months later & 61.66 & 16.23 & 26.34 & -2.9 & -3.0 \\
\hline 25 & 68 & $\mathbf{F}$ & $6^{\text {th }}$ months later & 85.83 & 1.73 & 57.87 & -4.5 & -3.9 \\
\hline 26 & 88 & $\mathbf{F}$ & $6^{\text {th }}$ months later & 42.3 & 19.94 & 31.85 & -3.9 & -4.0 \\
\hline 27 & 74 & $\mathbf{F}$ & $6^{\text {th }}$ months later & 74.15 & 4.79 & 38.23 & -3.6 & -3.8 \\
\hline 28 & 86 & $\mathbf{F}$ & $6^{\text {th }}$ months later & 40.33 & 20.77 & 18.54 & -4.9 & -4.7 \\
\hline
\end{tabular}




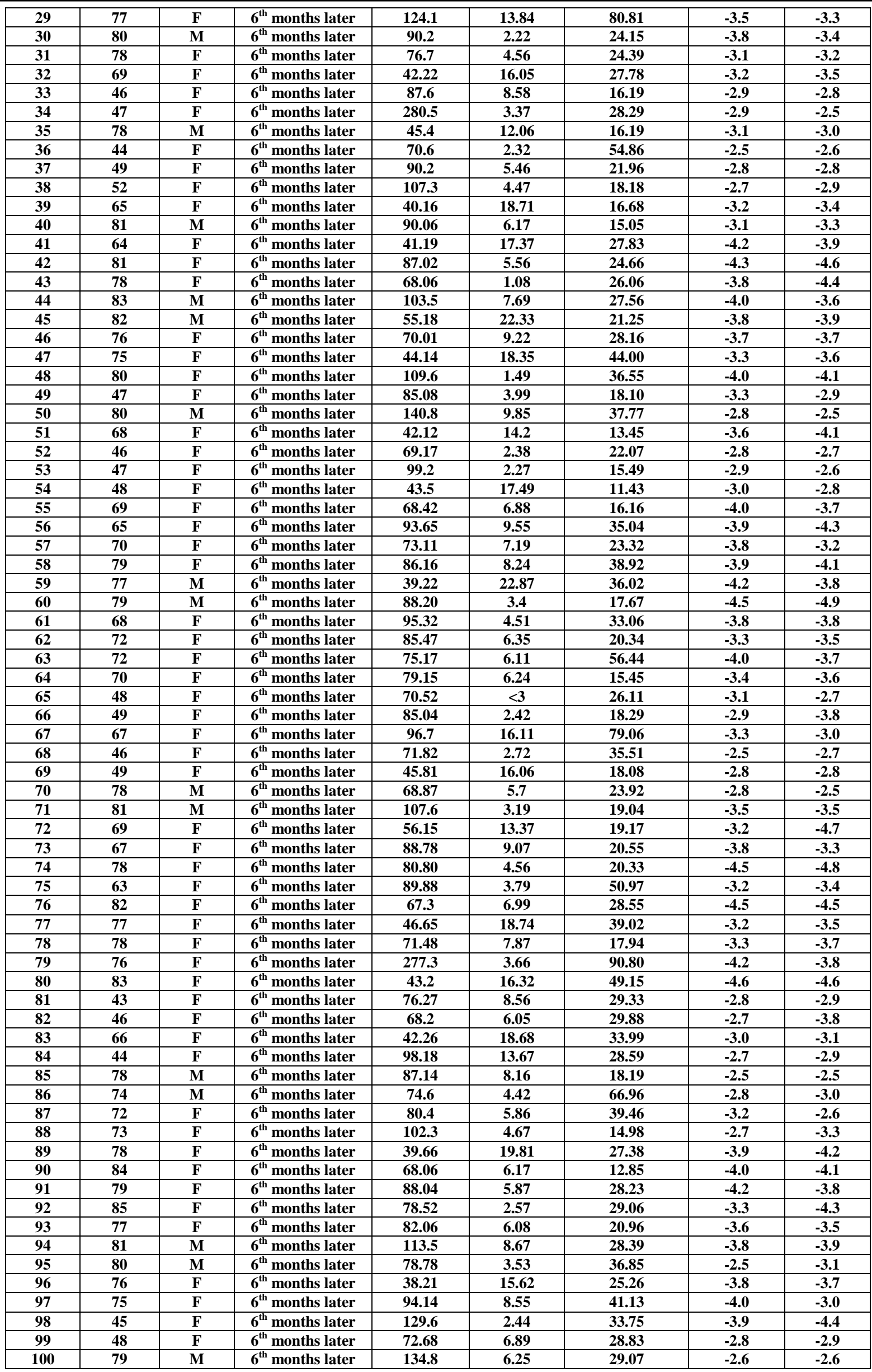


Table 4: 12 months post therapy

\begin{tabular}{|c|c|c|c|c|c|c|c|c|}
\hline \multirow[t]{2}{*}{ No } & \multirow[t]{2}{*}{ Age } & \multirow[t]{2}{*}{ Sex } & \multirow[t]{2}{*}{$\begin{array}{c}\text { Time of } \\
\text { Presentation } \\
(12 \text { months later })\end{array}$} & \multicolumn{3}{|c|}{ Biomarkers } & \multicolumn{2}{|c|}{$\begin{array}{c}\text { BMD } \\
\text { Scan / T Score }\end{array}$} \\
\hline & & & & PTH & VIT D & Osteocalcin & Lumbar & Femur \\
\hline 1 & 52 & $\mathbf{F}$ & 12 months later & 60.22 & 15.3 & 22.65 & -2.7 & -2.6 \\
\hline 2 & 54 & $\mathbf{F}$ & 12 months later & 83.11 & 3.34 & 29.59 & -2.5 & -2.7 \\
\hline 3 & 56 & $\mathbf{F}$ & 12 months later & 62.7 & 13.22 & $\mathbf{1 7 . 5 5}$ & -4.2 & -3.9 \\
\hline 4 & 53 & $\mathbf{F}$ & 12 months later & 60.11 & 18.69 & 14.69 & -3.1 & -3.0 \\
\hline 5 & 77 & $\mathbf{M}$ & 12 months later & 63.45 & 17.99 & 19.19 & -2.8 & -2.8 \\
\hline 6 & 82 & $\mathbf{F}$ & 12 months later & 61.55 & 19.75 & 38.34 & -2.6 & -2.5 \\
\hline 7 & 66 & $\mathbf{F}$ & 12 months later & 60.16 & 19.14 & 27.88 & -3.2 & -3.9 \\
\hline 8 & 63 & $\mathbf{F}$ & 12 months later & 93.96 & 4.53 & 30.04 & -3.1 & -3.3 \\
\hline 9 & 76 & $\mathbf{F}$ & 12 months later & 52.99 & 12.77 & 38.22 & -4.9 & -3.9 \\
\hline 10 & 83 & $\mathbf{M}$ & 12 months later & 60.12 & 13.12 & 15.97 & -4.6 & -4.8 \\
\hline 11 & 79 & M & 12 months later & 61.2 & 18.56 & 26.06 & -3.1 & -3.0 \\
\hline 12 & 59 & $\mathbf{F}$ & 12 months later & 53.44 & 20.55 & 20.04 & -3.8 & -3.9 \\
\hline 13 & 56 & $\mathbf{F}$ & 12 months later & 88.7 & 6.46 & 21.57 & -3.2 & -3.1 \\
\hline 14 & 62 & $\mathbf{F}$ & 12 months later & 51.45 & 19.64 & 18.54 & -3.9 & -3.5 \\
\hline 15 & 64 & $\mathbf{F}$ & 12 months later & 54.23 & 9.22 & 16.11 & -4.2 & -4.0 \\
\hline 16 & 74 & $\mathbf{F}$ & 12 months later & 99.05 & 2.46 & 161.3 & -4.3 & -4.7 \\
\hline 17 & 53 & $\mathbf{F}$ & 12 months later & 87.15 & 16.11 & 85.66 & -3.2 & -2.9 \\
\hline 18 & 48 & $\mathbf{F}$ & 12 months later & 58.82 & 17.72 & 36.01 & -3.3 & -2.8 \\
\hline 19 & 57 & $\mathbf{F}$ & 12 months later & 50.88 & 16.06 & 17.18 & -3.8 & -3.6 \\
\hline 20 & 46 & $\mathbf{M}$ & 12 months later & 52.87 & 15.7 & 21.92 & -2.9 & -2.7 \\
\hline 21 & 49 & $\mathbf{F}$ & 12 months later & 49.16 & 14.19 & 19.04 & -3.8 & -4.2 \\
\hline 22 & 45 & $\mathbf{F}$ & 12 months later & 48.20 & 13.37 & 11.07 & -2.6 & -2.8 \\
\hline 23 & 65 & $\mathbf{F}$ & 12 months later & 56.69 & 14.04 & 24.35 & -3.8 & -4.1 \\
\hline 24 & 64 & $\mathbf{F}$ & 12 months later & 44.66 & 16.76 & 27.94 & -2.9 & -3.0 \\
\hline 25 & 68 & $\mathbf{F}$ & 12 months later & 55.33 & 17.22 & 59.47 & -4.5 & -3.9 \\
\hline 26 & 88 & $\mathbf{F}$ & 12 months later & 48.3 & 14.94 & 21.25 & -3.9 & -4.0 \\
\hline 27 & 74 & $\mathbf{F}$ & 12 months later & 47.55 & 14.79 & 39.03 & -3.6 & -3.8 \\
\hline 28 & 86 & $\mathbf{F}$ & 12 months later & 46.47 & 16.87 & 14.04 & -4.2 & -4.0 \\
\hline 29 & 77 & $\mathbf{F}$ & 12 months later & 78.11 & 20.44 & 92.81 & -3.5 & -3.3 \\
\hline 30 & 80 & $\mathbf{M}$ & 12 months later & 90.2 & 2.22 & 24.15 & -3.8 & -3.4 \\
\hline 31 & 78 & $\mathbf{F}$ & 12 months later & 76.7 & 4.56 & 29.43 & -3.1 & -3.2 \\
\hline 32 & 69 & $\mathbf{F}$ & 12 months later & 42.22 & 16.05 & 28.88 & -3.2 & -3.5 \\
\hline 33 & 46 & $\mathbf{F}$ & 12 months later & 87.6 & 8.58 & 16.19 & -2.9 & -2.8 \\
\hline 34 & 47 & $\mathbf{F}$ & 12 months later & 280.5 & 3.37 & 27.09 & -2.9 & -2.5 \\
\hline 35 & 78 & $\mathbf{M}$ & 12 months later & 45.4 & 12.06 & 13.29 & -3.1 & -3.0 \\
\hline 36 & 44 & $\mathbf{F}$ & 12 months later & 70.6 & 2.32 & 58.86 & -2.5 & -2.5 \\
\hline 37 & 49 & $\mathbf{F}$ & 12 months later & 55.12 & 15.46 & 29.86 & -2.8 & -2.8 \\
\hline 38 & 52 & $\mathbf{F}$ & 12 months later & 107.3 & 4.47 & 15.08 & -2.7 & -2.9 \\
\hline 39 & 65 & $\mathbf{F}$ & 12 months later & 40.16 & 18.71 & 19.88 & -3.2 & -3.4 \\
\hline 40 & 81 & $\mathbf{M}$ & 12 months later & 90.06 & 6.17 & 18.05 & -3.1 & -3.3 \\
\hline 41 & 64 & $\mathbf{F}$ & 12 months later & 41.19 & 17.37 & 28.93 & -4.2 & -3.9 \\
\hline 42 & 81 & $\mathbf{F}$ & 12 months later & 61.02 & 11.56 & 27.96 & -4.3 & -4.6 \\
\hline 43 & 78 & $\mathbf{F}$ & 12 months later & 68.06 & $\mathbf{1 . 0 8}$ & 28.06 & -3.2 & -3.9 \\
\hline 44 & 83 & $\mathbf{M}$ & 12 months later & 103.5 & 7.69 & 20.36 & -4.0 & -3.6 \\
\hline 45 & 82 & $\mathbf{M}$ & 12 months later & 55.18 & 22.33 & 29.15 & -3.8 & -3.9 \\
\hline 46 & 76 & $\mathbf{F}$ & 12 months later & 57.11 & 19.82 & 20.06 & -3.7 & -3.7 \\
\hline 47 & 75 & $\mathbf{F}$ & 12 months later & 44.14 & 18.35 & 45.00 & -3.3 & -3.6 \\
\hline 48 & 80 & $\mathbf{F}$ & 12 months later & 109.6 & 1.49 & 37.55 & -4.0 & -4.1 \\
\hline 49 & 47 & $\mathbf{F}$ & 12 months later & 85.08 & 3.99 & 16.90 & -3.3 & -2.9 \\
\hline 50 & 80 & M & 12 months later & 140.8 & 9.85 & 31.77 & -2.8 & -2.5 \\
\hline 51 & 68 & $\mathbf{F}$ & 12 months later & 42.12 & 14.2 & 11.95 & -3.6 & -4.1 \\
\hline 52 & 46 & $\mathbf{F}$ & 12 months later & 62.07 & 10.99 & 22.77 & -2.8 & -2.7 \\
\hline 53 & 47 & $\mathbf{F}$ & 12 months later & 56.12 & 17.47 & 18.59 & -2.9 & -2.6 \\
\hline 54 & 48 & $\mathbf{F}$ & 12 months later & 43.5 & 17.49 & 16.73 & -3.0 & -2.8 \\
\hline 55 & 69 & $\mathbf{F}$ & 12 months later & 68.42 & 6.88 & 19.26 & -4.0 & -3.7 \\
\hline 56 & 65 & $\mathbf{F}$ & 12 months later & 93.65 & 9.55 & 38.14 & -3.9 & -4.3 \\
\hline 57 & 70 & $\mathbf{F}$ & 12 months later & 73.11 & 7.19 & 29.01 & -3.8 & -3.2 \\
\hline 58 & 79 & $\mathbf{F}$ & 12 months later & 86.16 & 8.24 & 30.42 & -3.9 & -4.1 \\
\hline 59 & 77 & $\mathbf{M}$ & 12 months later & 39.22 & 22.87 & 30.02 & -4.2 & -3.8 \\
\hline 60 & 79 & $\mathbf{M}$ & 12 months later & 88.20 & 3.4 & 19.77 & -4.5 & -4.9 \\
\hline 61 & 68 & $\mathbf{F}$ & 12 months later & 95.32 & 4.51 & 38.86 & -3.8 & -3.8 \\
\hline 62 & 72 & $\mathbf{F}$ & 12 months later & 85.47 & 6.35 & 29.64 & -3.3 & -3.5 \\
\hline 63 & 72 & $\mathbf{F}$ & 12 months later & 64.77 & 11.11 & 58.54 & -3.7 & -3.1 \\
\hline 64 & 70 & $\mathbf{F}$ & 12 months later & 79.15 & 6.24 & 11.55 & -3.4 & -3.6 \\
\hline 65 & 48 & $\mathbf{F}$ & 12 months later & 50.22 & 9.33 & 16.15 & -3.1 & -2.7 \\
\hline 66 & 49 & $\mathbf{F}$ & 12 months later & 85.04 & 2.42 & 18.93 & -2.9 & -3.8 \\
\hline 67 & 67 & $\mathbf{F}$ & 12 months later & 66.17 & 16.11 & 78.06 & -3.3 & -3.0 \\
\hline
\end{tabular}




\begin{tabular}{|c|c|c|c|c|c|c|c|c|}
\hline 68 & 46 & $\mathbf{F}$ & 12 months later & 64.82 & 10.72 & 33.01 & -2.5 & -2.7 \\
\hline 69 & 49 & $\mathbf{F}$ & 12 months later & 45.81 & 16.06 & 19.08 & -2.8 & -2.8 \\
\hline 70 & 78 & $\mathbf{M}$ & 12 months later & 68.87 & 5.7 & 27.92 & -2.8 & -2.5 \\
\hline 71 & 81 & $\mathbf{M}$ & 12 months later & 107.6 & 3.19 & 19.44 & -3.5 & -3.5 \\
\hline 72 & 69 & $\mathbf{F}$ & 12 months later & 56.15 & 13.37 & 18.07 & -3.0 & -4.0 \\
\hline 73 & 67 & $\mathbf{F}$ & 12 months later & 51.88 & 19.17 & 23.45 & -3.8 & -3.3 \\
\hline 74 & 78 & $\mathbf{F}$ & 12 months later & 80.80 & 4.56 & 24.03 & -4.5 & -4.8 \\
\hline 75 & 63 & $\mathbf{F}$ & 12 months later & 89.88 & 3.79 & 55.67 & -3.2 & -3.4 \\
\hline 76 & 82 & $\mathbf{F}$ & 12 months later & 63.13 & 10.99 & 27.65 & -4.5 & -4.5 \\
\hline 77 & 77 & $\mathbf{F}$ & 12 months later & 46.65 & 16.74 & 37.92 & -3.2 & -3.5 \\
\hline 78 & 78 & $\mathbf{F}$ & 12 months later & 71.48 & 7.87 & 19.94 & -3.3 & -3.7 \\
\hline 79 & 76 & $\mathbf{F}$ & 12 months later & 277.3 & 3.66 & 90.80 & -3.9 & -3.2 \\
\hline 80 & 83 & $\mathbf{F}$ & 12 months later & 43.2 & 16.32 & 48.55 & -4.6 & -4.6 \\
\hline 81 & 43 & $\mathbf{F}$ & 12 months later & 76.27 & 8.56 & 23.23 & -2.8 & -2.9 \\
\hline 82 & 46 & $\mathbf{F}$ & 12 months later & 64.12 & 10.05 & 26.88 & -2.7 & -3.8 \\
\hline 83 & 66 & $\mathbf{F}$ & 12 months later & 42.26 & 18.68 & 35.99 & -3.0 & -3.1 \\
\hline 84 & 44 & $\mathbf{F}$ & 12 months later & 61.28 & 19.67 & 28.99 & -2.7 & -2.9 \\
\hline 85 & 78 & $\mathbf{M}$ & 12 months later & 49.44 & 18.66 & 19.09 & -2.5 & -2.5 \\
\hline 86 & 74 & $\mathbf{M}$ & 12 months later & 74.6 & 4.42 & 69.76 & -2.8 & -3.0 \\
\hline 87 & 72 & $\mathbf{F}$ & 12 months later & 80.4 & 5.86 & 30.06 & -2.8 & -2.5 \\
\hline 88 & 73 & $\mathbf{F}$ & 12 months later & 102.3 & 4.67 & 14.98 & -2.7 & -3.3 \\
\hline 89 & 78 & $\mathbf{F}$ & 12 months later & 39.66 & 19.81 & 28.98 & -3.9 & -4.2 \\
\hline 90 & 84 & $\mathbf{F}$ & 12 months later & 63.06 & 10.17 & 19.95 & -4.0 & -4.1 \\
\hline 91 & 79 & $\mathbf{F}$ & 12 months later & 88.04 & 5.87 & 29.83 & -4.2 & -3.8 \\
\hline 92 & 85 & $\mathbf{F}$ & 12 months later & 78.52 & 2.57 & 29.06 & -3.3 & -4.3 \\
\hline 93 & 77 & $\mathbf{F}$ & 12 months later & 82.06 & 6.08 & 23.46 & -3.6 & -3.5 \\
\hline 94 & 81 & $\mathbf{M}$ & 12 months later & 113.5 & 8.67 & 20.09 & -3.3 & -3.1 \\
\hline 95 & 80 & $\mathbf{M}$ & 12 months later & 78.78 & $\mathbf{3 . 5 3}$ & 39.25 & -2.5 & -3.1 \\
\hline 96 & 76 & $\mathbf{F}$ & 12 months later & 38.21 & 15.62 & 23.96 & -3.8 & -3.7 \\
\hline 97 & 75 & $\mathbf{F}$ & 12 months later & 94.14 & 8.55 & 48.03 & -4.0 & -3.0 \\
\hline 98 & 45 & $\mathbf{F}$ & 12 months later & 55.00 & $\mathbf{1 7 . 2 2}$ & 39.45 & -3.9 & -4.4 \\
\hline 99 & 48 & $\mathbf{F}$ & 12 months later & 72.68 & 6.89 & 28.03 & -2.8 & -2.9 \\
\hline 100 & 79 & $\mathbf{M}$ & 12 months later & 134.8 & 6.25 & 24.97 & -2.6 & -2.6 \\
\hline
\end{tabular}

Table 5: 24 months post therapy

\begin{tabular}{|c|c|c|c|c|c|c|c|c|}
\hline \multirow[t]{2}{*}{ No } & \multirow[t]{2}{*}{ Age } & \multirow[t]{2}{*}{ Sex } & \multirow[t]{2}{*}{$\begin{array}{c}\text { Time of Presentation } \\
\left(24^{\text {th }} \text { months later }\right)\end{array}$} & \multicolumn{3}{|c|}{ Biomarkers } & \multicolumn{2}{|c|}{$\begin{array}{c}\text { BMD } \\
\text { Scan / T Score }\end{array}$} \\
\hline & & & & PTH & VIT D & Osteocalcin & Lumbar & Femur \\
\hline 1 & 52 & $\mathbf{F}$ & 24 months later & 59.12 & 21.13 & 11.05 & -2.3 & -2.2 \\
\hline 2 & 54 & $\mathbf{F}$ & 24 months later & 60.51 & 13.34 & 22.99 & -2.5 & -2.7 \\
\hline $\mathbf{3}$ & 56 & $\mathbf{F}$ & 24 months later & 51.17 & 23.12 & 18.75 & -4.2 & -3.9 \\
\hline 4 & 53 & $\mathbf{F}$ & 24 months later & 52.41 & 26.99 & 16.99 & -2.4 & -2.1 \\
\hline 5 & 77 & $\mathbf{M}$ & 24 months later & 48.55 & 31.19 & 15.04 & -2.8 & -2.8 \\
\hline 6 & 82 & $\mathbf{F}$ & 24 months later & 49.35 & 29.55 & 33.44 & -2.1 & -2.0 \\
\hline 7 & 66 & $\mathbf{F}$ & 24 months later & 52.66 & 20.33 & 26.98 & -2.4 & -2.6 \\
\hline 8 & 63 & $\mathbf{F}$ & 24 months later & 62.66 & 14.53 & 39.84 & -3.1 & $\begin{array}{l}-3.3 \\
\end{array}$ \\
\hline 9 & 76 & $\mathbf{F}$ & 24 months later & 42.49 & 32.27 & 36.02 & -4.9 & $\begin{array}{l}-3.9 \\
\end{array}$ \\
\hline 10 & 83 & $\mathbf{M}$ & 24 months later & 44.32 & 23.22 & 18.17 & -2.3 & -2.4 \\
\hline 11 & 79 & $\mathbf{M}$ & 24 months later & 42.12 & 33.66 & 20.06 & -3.1 & $\begin{array}{l}-3.0 \\
-3.0\end{array}$ \\
\hline 12 & 59 & $\mathbf{F}$ & 24 months later & 43.14 & 30.45 & 21.04 & -2.8 & -2.9 \\
\hline 13 & 56 & $\mathbf{F}$ & 24 months later & 59.17 & 16.34 & 22.57 & -3.2 & -3.1 \\
\hline 14 & 62 & $\mathbf{F}$ & 24 months later & 41.55 & 28.44 & 18.44 & -3.9 & -3.5 \\
\hline 15 & 64 & $\mathbf{F}$ & 24 months later & 44.13 & 29.32 & 21.31 & -2.7 & -2.2 \\
\hline 16 & 74 & $\mathbf{F}$ & 24 months later & 51.15 & 12.03 & 181.3 & -4.3 & -4.7 \\
\hline 17 & 53 & $\mathbf{F}$ & 24 months later & $\mathbf{5 3 . 1 5}$ & 26.31 & 66.66 & -3.2 & -2.9 \\
\hline 18 & 48 & $\mathbf{F}$ & 24 months later & 41.22 & 27.52 & 38.01 & -2.3 & -2.4 \\
\hline 19 & 57 & $\mathbf{F}$ & 24 months later & 40.68 & 26.16 & 19.08 & -3.8 & -3.6 \\
\hline 20 & 46 & $\mathbf{M}$ & 24 months later & 43.77 & 28.33 & 20.02 & -2.9 & -2.7 \\
\hline 21 & 49 & $\mathbf{F}$ & 24 months later & 41.76 & 34.79 & 14.44 & -2.3 & -2.8 \\
\hline 22 & 45 & $\mathbf{F}$ & 24 months later & 34.30 & 35.77 & 17.77 & -2.0 & -1.8 \\
\hline 23 & 65 & $\mathbf{F}$ & 24 months later & 41.99 & 29.14 & 25.85 & -3.8 & -4.1 \\
\hline 24 & 64 & $\mathbf{F}$ & 24 months later & 39.16 & 34.66 & 20.84 & -2.1 & -2.0 \\
\hline 25 & 68 & $\mathbf{F}$ & 24 months later & 38.13 & 36.12 & $\mathbf{5 7 . 7 7}$ & -4.5 & -3.9 \\
\hline 26 & 88 & $\mathbf{F}$ & 24 months later & 39.13 & 37.44 & 22.25 & -3.9 & -4.0 \\
\hline 27 & 74 & $\mathbf{F}$ & 24 months later & 42.15 & 26.69 & 32.23 & -3.6 & -3.8 \\
\hline 28 & 86 & $\mathbf{F}$ & 24 months later & 40.77 & 32.67 & 18.54 & -3.0 & -2.8 \\
\hline 29 & 77 & $\mathbf{F}$ & 24 months later & 58.14 & 31.64 & 99.11 & -3.5 & -3.3 \\
\hline 30 & 80 & $\mathbf{M}$ & 24 months later & 56.12 & 12.52 & 28.55 & -2.0 & -2.1 \\
\hline
\end{tabular}




\begin{tabular}{|c|c|c|c|c|c|c|c|c|}
\hline 31 & 78 & $\mathbf{F}$ & 24 months later & 76.7 & 4.56 & 29.13 & -2.5 & -2.3 \\
\hline 32 & 69 & $\mathbf{F}$ & 24 months later & 38.62 & 26.25 & 27.88 & -3.2 & -3.5 \\
\hline 33 & 46 & $\mathbf{F}$ & 24 months later & 45.16 & 18.88 & 15.09 & -2.9 & -2.8 \\
\hline 34 & 47 & $\mathbf{F}$ & 24 months later & 60.15 & 16.77 & 25.99 & -2.9 & -2.5 \\
\hline 35 & 78 & M & 24 months later & 39.14 & 27.06 & 17.69 & -3.1 & -3.0 \\
\hline 36 & 44 & $\mathbf{F}$ & 24 months later & 54.16 & 16.32 & 55.66 & -2.1 & -2.0 \\
\hline 37 & 49 & $\mathbf{F}$ & 24 months later & 45.22 & 21.66 & 28.06 & -2.8 & -2.8 \\
\hline 38 & 52 & $\mathbf{F}$ & 24 months later & 99.13 & 7.47 & 18.18 & -2.7 & -2.9 \\
\hline 39 & 65 & $\mathbf{F}$ & 24 months later & 36.66 & 28.11 & 17.88 & -2.0 & -2.2 \\
\hline 40 & 81 & M & 24 months later & 59.26 & 16.17 & 16.15 & -3.1 & -3.3 \\
\hline 41 & 64 & $\mathbf{F}$ & 24 months later & 35.29 & 27.17 & 28.03 & -4.2 & -3.9 \\
\hline 42 & 81 & $\mathbf{F}$ & 24 months later & 87.02 & 5.56 & 28.16 & -4.3 & -4.6 \\
\hline 43 & 78 & $\mathbf{F}$ & 24 months later & 57.26 & 23.28 & 26.36 & -2.0 & -2.2 \\
\hline 44 & 83 & M & 24 months later & 60.15 & 17.69 & 25.96 & -4.0 & -3.6 \\
\hline 45 & 82 & M & 24 months later & 42.28 & 22.13 & 22.15 & -3.8 & -3.9 \\
\hline 46 & 76 & $\mathbf{F}$ & 24 months later & 46.31 & 27.12 & 28.96 & -3.7 & -3.7 \\
\hline 47 & 75 & $\mathbf{F}$ & 24 months later & 42.44 & 28.55 & 48.00 & -3.3 & -3.6 \\
\hline 48 & 80 & $\mathbf{F}$ & 24 months later & 61.16 & 19.89 & 35.35 & -2.0 & -2.2 \\
\hline 49 & 47 & $\mathbf{F}$ & 24 months later & 85.08 & 3.99 & 18.90 & -3.3 & -2.9 \\
\hline 50 & 80 & M & 24 months later & 60.18 & 19.55 & 35.77 & -2.8 & -2.5 \\
\hline 51 & 68 & $\mathbf{F}$ & 24 months later & 42.12 & 21.12 & 10.45 & -3.6 & -4.1 \\
\hline 52 & 46 & $\mathbf{F}$ & 24 months later & 58.77 & $\mathbf{1 7 . 8 8}$ & 22.87 & -2.3 & -2.1 \\
\hline 53 & 47 & $\mathbf{F}$ & 24 months later & 46.22 & 27.47 & 18.99 & -2.9 & -2.6 \\
\hline 54 & 48 & $\mathbf{F}$ & 24 months later & 43.5 & 27.49 & 18.93 & -3.0 & -2.8 \\
\hline 55 & 69 & $\mathbf{F}$ & 24 months later & 49.22 & 16.78 & 18.46 & -3.0 & -2.8 \\
\hline 56 & 65 & $\mathbf{F}$ & 24 months later & 93.65 & 9.55 & 30.54 & -3.9 & -4.3 \\
\hline 57 & 70 & $\mathbf{F}$ & 24 months later & 46.33 & 17.29 & 28.81 & -3.8 & -3.2 \\
\hline 58 & 79 & $\mathbf{F}$ & 24 months later & 86.16 & 8.24 & 38.92 & -2.4 & -2.3 \\
\hline 59 & 77 & M & 24 months later & 39.33 & 22.77 & 38.22 & -4.2 & -3.8 \\
\hline 60 & 79 & $\mathbf{M}$ & 24 months later & 59.12 & 16.14 & 18.77 & -4.5 & -4.9 \\
\hline 61 & 68 & $\mathbf{F}$ & 24 months later & 95.32 & 4.51 & 39.76 & -2.2 & -2.1 \\
\hline 62 & 72 & $\mathbf{F}$ & 24 months later & 85.47 & 6.35 & 25.44 & -3.3 & -3.5 \\
\hline 63 & 72 & $\mathbf{F}$ & 24 months later & 55.17 & 16.11 & 58.84 & -2.0 & -1.9 \\
\hline 64 & 70 & $\mathbf{F}$ & 24 months later & 49.55 & 16.24 & 14.55 & -3.4 & -3.6 \\
\hline 65 & 48 & $\mathbf{F}$ & 24 months later & 40.12 & 19.23 & 26.95 & -3.1 & -2.7 \\
\hline 66 & 49 & $\mathbf{F}$ & 24 months later & 51.24 & 17.22 & 19.23 & -1.8 & -2.0 \\
\hline 67 & 67 & $\mathbf{F}$ & 24 months later & 49.37 & 24.41 & 65.96 & -3.3 & -3.0 \\
\hline 68 & 46 & $\mathbf{F}$ & 24 months later & 52.22 & 32.32 & 33.30 & -1.7 & -1.5 \\
\hline 69 & 49 & $\mathbf{F}$ & 24 months later & 45.11 & 26.36 & 14.18 & -2.8 & -2.8 \\
\hline 70 & 78 & M & 24 months later & 43.67 & 15.47 & 21.02 & -2.2 & -2.4 \\
\hline 71 & 81 & M & 24 months later & 107.6 & 3.19 & 15.84 & -3.5 & -3.5 \\
\hline 72 & 69 & $\mathbf{F}$ & 24 months later & 46.35 & 23.77 & 18.07 & -1.2 & -1.2 \\
\hline 73 & 67 & $\mathbf{F}$ & 24 months later & 41.88 & 29.17 & 25.85 & -3.8 & -3.3 \\
\hline 74 & 78 & $\mathbf{F}$ & 24 months later & $\mathbf{5 0 . 0 0}$ & 17.66 & 21.03 & -4.5 & -4.8 \\
\hline 75 & 63 & $\mathbf{F}$ & 24 months later & 89.88 & 3.79 & $\mathbf{5 0 . 0 7}$ & -3.2 & -3.4 \\
\hline 76 & 82 & $\mathbf{F}$ & 24 months later & 44.00 & 16.19 & 20.25 & -3.2 & -3.4 \\
\hline 77 & 77 & $\mathbf{F}$ & 24 months later & 46.65 & 18.74 & 30.02 & -3.2 & -3.5 \\
\hline 78 & 78 & $\mathbf{F}$ & 24 months later & 71.48 & 7.87 & 19.74 & -3.3 & -3.7 \\
\hline 79 & 76 & $\mathbf{F}$ & 24 months later & 167.3 & 7.66 & 99.70 & -1.3 & -1.8 \\
\hline 80 & 83 & $\mathbf{F}$ & 24 months later & 43.2 & 26.32 & 44.55 & -4.6 & -4.6 \\
\hline 81 & 43 & $\mathbf{F}$ & 24 months later & 46.77 & 18.56 & 26.53 & -2.8 & -2.9 \\
\hline 82 & 46 & $\mathbf{F}$ & 24 months later & 68.2 & 6.05 & 28.98 & -2.7 & -3.8 \\
\hline 83 & 66 & $\mathbf{F}$ & 24 months later & 42.26 & 28.68 & 39.59 & -2.7 & -2.8 \\
\hline 84 & 44 & $\mathbf{F}$ & 24 months later & 41.78 & 28.00 & 21.09 & -2.7 & -2.9 \\
\hline 85 & 78 & M & 24 months later & 49.44 & 28.66 & 18.59 & -2.0 & -1.8 \\
\hline 86 & 74 & $\mathbf{M}$ & 24 months later & 42.16 & 14.22 & 60.06 & -2.8 & -3.0 \\
\hline 87 & 72 & $\mathbf{F}$ & 24 months later & 80.4 & 5.86 & 34.96 & -1.6 & -1.8 \\
\hline 88 & 73 & $\mathbf{F}$ & 24 months later & 102.3 & 4.67 & 18.88 & -2.7 & -3.3 \\
\hline 89 & 78 & $\mathbf{F}$ & 24 months later & 39.66 & 27.11 & 24.18 & -3.9 & -4.2 \\
\hline 90 & 84 & $\mathbf{F}$ & 24 months later & 48.00 & 16.10 & 18.05 & -2.2 & -2.0 \\
\hline 91 & 79 & $\mathbf{F}$ & 24 months later & 88.04 & 5.87 & 29.03 & -4.2 & -3.8 \\
\hline 92 & 85 & $\mathbf{F}$ & 24 months later & 43.02 & 19.00 & 27.96 & -3.3 & -4.3 \\
\hline 93 & 77 & $\mathbf{F}$ & 24 months later & 82.06 & 6.08 & 25.66 & -3.6 & -3.5 \\
\hline 94 & 81 & $\mathbf{M}$ & 24 months later & 113.5 & 8.67 & 24.81 & -2.0 & -1.6 \\
\hline 95 & 80 & M & 24 months later & 78.78 & $\mathbf{3 . 5 3}$ & 37.05 & -2.5 & -3.1 \\
\hline 96 & 76 & $\mathbf{F}$ & 24 months later & 38.00 & 25.00 & 23.96 & -3.8 & -3.7 \\
\hline 97 & 75 & $\mathbf{F}$ & 24 months later & 94.14 & 8.55 & 48.93 & -1.9 & -1.1 \\
\hline 98 & 45 & $\mathbf{F}$ & 24 months later & 44.00 & 27.02 & 34.85 & -3.9 & -4.4 \\
\hline 99 & 48 & $\mathbf{F}$ & 24 months later & 72.68 & 6.89 & 27.13 & -2.5 & -2.6 \\
\hline 100 & 79 & $\mathbf{M}$ & 24 months later & 134.8 & 6.25 & 28.77 & -2.1 & -2.0 \\
\hline
\end{tabular}




\section{JMSCR Vol||06||Issue||03||Page 827-840||March}

We detected the Dexa scan Absorptiometry are superior \& more sensitive from the index data of same 100 Osteoporotic patients in early diagnosis $100 \%$ compared to bone biomarkers in diagnostic fact which is reach to $85 \%$. Also, we detected $33 \%$ after 24 months in post therapy treatment follow up for Dexa scan Compare to $78 \%$ in bone biomarkers to same patients, also for the same follow up period rates times, which is more sensitive index scores in osteoporotic patients.

Table 6: Total changes $24^{\text {th }}$ months Dexa Scan \& Blood Biomarkers

\begin{tabular}{|c|c|c|}
\hline & Dexa Scan & Blood Biomarkers \\
\hline No. of patients & 100 & 100 \\
\hline Early / Diagnosis & $100 \%$ & $85 \%$ \\
\hline 6 Months & $8 \%$ & $24 \%$ \\
\hline 12 Months & $11 \%$ & $28 \%$ \\
\hline Total changes \% / Data Changes 2 years & $33 \%$ & $78 \%$ \\
\hline P VALUE & 0.08 & $<0.0001$ \\
\hline
\end{tabular}

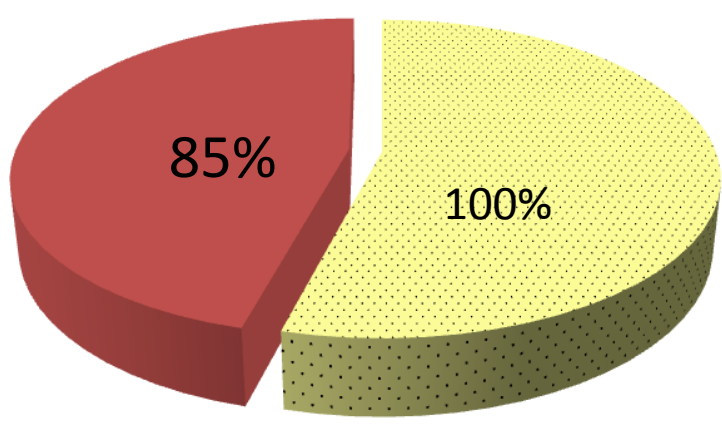

Dexa Scan

Blood Biomarkers

Pia chart: 100 patients Pia chart showing Dexa scan diagnostic compare to Blood Biomarkers early diagnosis in patients

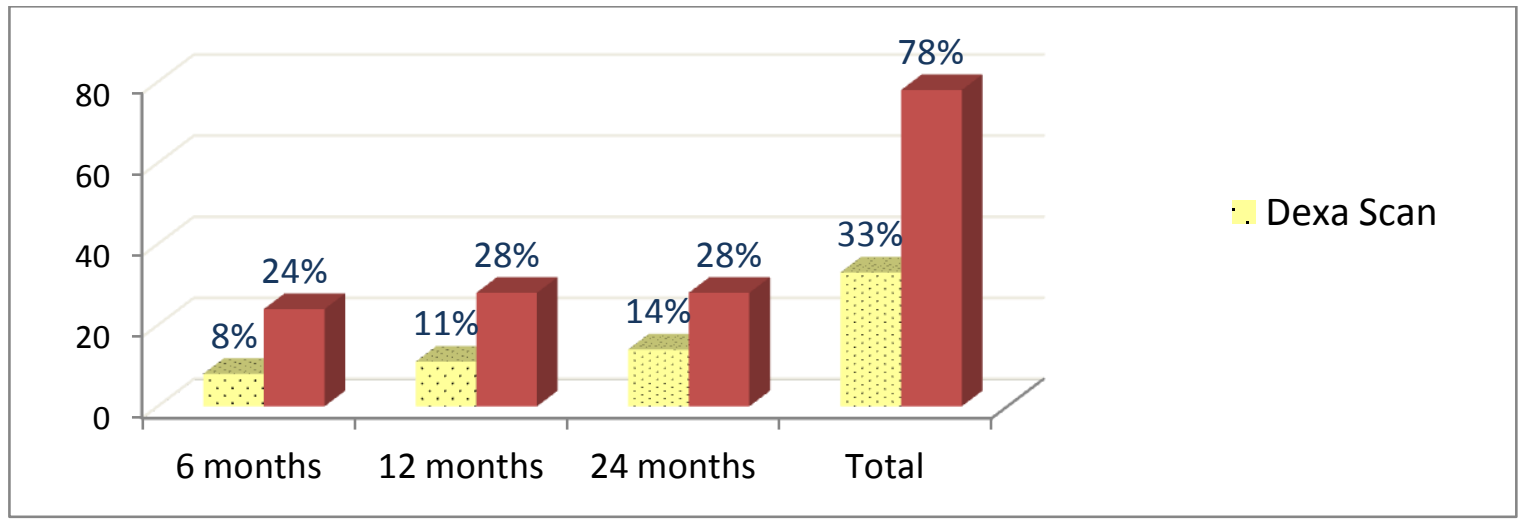

Figure 5: Post treatment follow up 


\section{Discussion}

Osteoporosis is a systemic disease characterized by low bone mass and microarchitectural deterioration of bone tissue, resulting in an increased risk of fracture. While the level of bone mass can be estimated by measuring bone mineral density (BMD) using dual X-ray absorptiometry (DXA), its measurement does not capture all the risk factors for fracture. Quantitative changes in skeletal turnover can be assessed easily and noninvasively by the measurement of serum and urinary biochemical markers; the most sensitive markers include serum osteocalcin, bone specific alkaline phosphatase, Vitamin D3, Parathyroid Hormone PTH assessments.

MICHAEL P. J.etal, support our study, they recommend using dual energy x-ray absorptiometry to screen all women 65 years and older evidence to recommend screening for osteoporosis in patients with newly diagnosed osteoporosis, suggested laboratory tests to identify secondary causes include serum 25hydroxyvitamin D, calcium, creatinine, and thyroid-stimulating hormone ${ }^{14,15,16,17,18}$. After initiation of treatment, the need for follow-up bone density testing is uncertain. These confirmed our results data requirements of Bone Biomarkers as diagnostic factors during follow up treatments. these supportive ideas confirm our study results data $^{19,20}$.WHOWorld Health Organization. Assessment of fracture risk osteoporotic patients, Kanis J., they evaluated other methods for diagnosing osteoporosis that have been used extensively in clinical trials and epidemiological studies. These include radiological assessments and Bone Turnover Markers (BTM). These supported our study to use the Dexa Scan Absorptiometry plus Bone Biomarkers both in diagnostic \& Therapeutic follow up of Osteoporotic patients with proper method of evaluation which is for diagnosis, plus which is for treatment follow up ${ }^{21,22}$. Combination BMD with BTM could improve fracture prediction in postmenopausal women. One advantage of biochemical markers compared to BMD is early estimation of treatment effect. Significant changes in BTM can be seen during antiresorptive therapy after a few weeks of treatment; whereas individual monitoring with DXA usually requires 1-2 years to identify significant changes. As adherence is an important issue of long-term therapy in chronic disease, it has been suggested that BTM could be used in clinical practice to assess the patient's adherence to treatment and also provide feedback on the effectiveness of the medication ${ }^{23,24,25,26}$. In Our study confirm the usage these two methods, theDexa Scan Absorptiometry \& Bone Biomarkersduring diagnostic criteria, plus evaluation during post therapeutic treatment follow up. The Dexa Scan Absorptiometry are more sensitive index in diagnostic determination, plus Bone Biomarkers method are superior in post therapeutic treatment follow up of osteoporotic patients.

\section{Conclusions}

We concluded that Dexa Scan Absorptiometry is superior in diagnosis of Osteoporosis than Bone Biomarkers evaluation in compared to Bone Biomarkers are superior in Therapeutic Post Treatment Monitoring for 2 years follow up of Osteoporotic Patients.

\section{References}

1. National Osteoporosis Foundation. America's Bone Health: The State of Osteoporosis and Low Bone Mass in Our Nation. Washington, DC: National Osteoporosis Foundation; 2002.

2. Moyad MA. Preventing male osteoporosis: prevalence, risks, diagnosis and imaging tests. Urol Clin N Am. 2004;31:321-330. doi: 10.1016/j.ucl.2004.01.008.

3. Neetakumar, Ammini AC, Tandon N, Goswami R, Dineshkumar, Singh A. Ethnic variation of host and risk factors in silent epidemic of osteoporosis. Orthoped Today. 2004;VI(4):240-244.

4. Kanis JA, Melton LJ III, Christiansen C, Johnston CC, Khaltaev N.The diagnosis of 
osteoporosis. J Bone Miner Res. 1994;9(8):1137-1141

5. Raisz LG. Physiology and pathophysiology of bone remodeling. Clin Chem. 1999;45:1353-1358.

6. Black D, Bilezikian J, Ensrud K, Greenspan S, Palermo L, Hue T, et al. One year of alendronate after one year of parathyroid hormone(1-84) for osteoporosis. N Engl J Med. 2005;353:555-565.

7. Garnero P, Darte C, Delmas P. A model to monitor the efficacy of alendronate treatment in women with osteoporosis using a biochemical marker of bone turnover. Bone. 1999;24:603-609.

8. Cremers S, Garnero P. Biochemical markers of bone turnover in the clinical development of drugs for osteoporosis and metastatic bone disease: potential uses and pitfalls. Drugs. 2006;66:2031-2058.

9. Charles P, Poser JW, Mosekilde L, Jensen FT. Estimation of bone turnover evaluated by $47 \mathrm{Ca}$-kinetics. Efficiency of serum bone gamma-carboxyglutamic acidcontaining protein, serum alkaline phosphatase, and urinary hydroxyproline excretion. Journal of Clinical Investigation. 1985;76:2254-2258.

10. Eriksen EF, Charles P, Melsen F, Mosekilde L, RisteliL, Risteli J. Serum markers of type I collagen formation and degradation in metabolic bone disease: correlation with bone histomorphometry. Journal of Bone Mineral Research. 1993;8:127-132.

11. R. Paul Robertson, MD, Sundeep Khosla, MD. Translational Endocrinology \& Metabolism. The endocrine society. Volume I, Number 1, 2010

12. Allison JL, Stephen H, Richard E. Measurement of osteocalcin. Ann Clin Biochem. 2000;37:432-446.

13. Swaminathan R. Biochemical markers of bone turnover. Clin Chim Acta. 2001;313:95-105.
14. National Osteoporosis Foundation. Clinician's Guide to Prevention and Treatment of Osteoporosis. Washington, DC: National Osteoporosis Foundation; 2014.

15. U.S. Department of Health and Human Services. Bone Health and Osteoporosis: A Report of the Surgeon General. Rockville, Md.: U.S. Department of Health and Human Services, Office of the Surgeon General; 2004.

16. National Osteoporosis Foundation. America's Bone Health: The State of Osteoporosis and Low Bone Mass in Our Nation. Washington, DC: National Osteoporosis Foundation; 2002.

17. World Health Organization. WHO scientific group on the assessment of osteoporosis at the primary health care level: summary meeting report. Brussels, Belgium; May 5-7, 2004. Geneva, Switzerland: World Health Organization; 2007.

18. U. S. Preventive Services Task Force. Screening for osteoporosis: U.S. Preventive Services Task Force recommendation statement. Ann Intern Med. 2011;154(5):356-364.

19. Lewiecki EM. Bone density monitoring to monitor osteoporosis therapy in clinical practice. Am Fam Physician. 2010;82 (7):749-754.

20. Combs BP, Rappaport M, Caverly TJ, Matlock DD. "Due" for a scan: examining the utility of monitoring densitometry. JAMA Intern Med. 2013;173(21):20072009.

21. World Health Organization. Assessment of fracture risk and its implication to screening for postmenopausal osteoporosis: Technical report series 843 . Geneva: WHO,1994

22. Kanis J. Diagnosis of osteoporosis and assessment of fracture risk. Lancet 2002;359:1929-36 
23. Garnero P. Markers of bone turnover for the prediction of fracture risk. Osteoporos Int. 2000;11 Suppl 6:S55-65

24. Delmas PD, Eastell P, Garnero P, Seibel MJ, Stepan J, Committee of Scientific Advisors of the International Osteoporosis Foundation. The use of biochemical markers of bone turnover in osteoporosis. Osteoporos Int. 2000;11 Suppl 6:S2-17.

25. Johnell O, Odén A, De LAet C, Garnero P, Delmas PD, Kanis JA. Biochemical indices of bone turnover and the assessment of fracture probability. Osteoporosis Int 2002;13:523-6

26. Delmas PD, Vrijens B, Roux C, LeMoigne-Amrani A,. Eastell R, Grauer A, Watts NB, Pols HA, Ringe JD, Cahall D. A Reinforcement Message Based on Bone Turnover Marker Response Influences Long-Term Persistence with Risedronate in Osteoporosis: The IMPACT Study.. ASBMR 2003 [Poster M330] 